\title{
Perfil dos Psicólogos Brasileiros que Utilizam Testes Psicológicos: Áreas e Instrumentos Utilizados
}

\author{
Caroline Tozzi Reppold ${ }^{1}$ \\ ${ }^{1}$ Universidade Federal de Ciências da Saúde de \\ Porto Alegre, RS, Brasil. \\ Solange Muglia Wechsler ${ }^{2}$ \\ ${ }^{2}$ Pontificia Universidade Católica de Campinas, \\ SP, Brasil.
}

\author{
Leandro da Silva Almeida ${ }^{3}$ \\ ${ }^{3}$ Universidade do Minho, Braga, Portugal. \\ Paula Elosua ${ }^{4}$ \\ ${ }^{4}$ Universidad del País Vasco, San Sebastian, Espanha. \\ Claudio Simon Hutz ${ }^{5}$ \\ ${ }^{5}$ Universidade Federal do Rio Grande do Sul, RS, Brasil.
}

\begin{abstract}
Resumo: O artigo teve como objetivo apresentar um levantamento sobre o perfil dos psicólogos brasileiros que utilizam testes psicológicos no que tange a sua(s) área(s) de atuação, ao uso que fazem dos instrumentos psicológicos em suas práticas e aos testes que utilizam com maior frequência no exercício profissional. Participaram do estudo 668 psicólogos, que exerciam a profissão, em média, a 14,06 anos ( $\mathrm{DP}=11,34)$. Os dados foram coletados em plataforma on-line, por meio de questionário que está sendo utilizado em vários países da América do Sul, divulgado a todos os psicólogos brasileiros com apoio de órgãos da classe. Os resultados obtidos evidenciaram que a Psicologia Clínica e da Saúde são as áreas mais prevalentes de atuação principal dos psicólogos brasileiros. Testes psicológicos são utilizados principalmente para fins psicodiagnósticos. Os grupos etários mais frequentemente cobertos pelas avaliações realizadas são adultos. Os testes psicológicos mais utilizados são destinados a avaliação de personalidade ou inteligência. A lista inclui Teste Palográfico, HTP, Rorschach, Bateria Fatorial de Personalidade e as Escalas Wechsler de Inteligência. Os testes projetivos/expressivos ou gráficos são os mais usuais para avaliação da personalidade. A maioria das respostas obtidas na análise dos cinco testes mais utilizados refere-se a algum teste considerado favorável para uso pelo Satepsi. Destaca-se a extensão da lista dos testes citados, o que indica que os psicólogos estão atentos aos novos instrumentos e às opções de que dispõem para qualificar as avaliações realizadas. Contudo, é grande o número de instrumentos utilizados que não têm avaliação certificada de suas propriedades técnicas.
\end{abstract}

Palavras-chave: Avaliação Psicológica, Testes Psicológicos, Instrumentos Psicológicos.

\section{Profile of Brazilian Psychologists Using Psychological Tests: Areas and Instruments Used}

\begin{abstract}
This article presents a survey on the profile of Brazilian psychologists who use psychological tests regarding their area(s) of action, their use of psychological instruments in their practices and the tests they use more frequently in their professional practice. A total of 668 psychologists who practiced the profession participated in this study, having average 14.06 years $(\mathrm{SD}=11.34)$. The data were collected with use of an online platform via a questionnaire that is being used in several countries of South America divulged to all Brazilian psychologists with the support of institutions related to the field. The results obtained evidenced that Clinical and Health Psychology are the most prevalent areas of action of Brazilian psychologists. Psychological tests
\end{abstract}


are mainly used for psychodiagnostic purposes. The age groups most frequently covered by the assessments are adults. The most commonly used psychological tests serve for the evaluation of personality or intelligence. The list includes Palographic Test, HTP, Rorschach, Factorial Personality Battery and the Wechsler Intelligence Scales. Projective/expressive or graphical tests are the most common for personality assessment. Most of the answers obtained in the analysis of the five most used tests refer to some test considered favorable for use by Satepsi. It is worth noting the list of cited tests, which indicates that psychologists are attentive to new instruments and the options available to qualify the evaluations performed. However, there is a large number of instruments used that have no certified evaluation of their technical properties.

Keywords: Psychological Assessment, Psychological Tests, Psychological Instruments.

\title{
Perfil de los Psicólogos Brasileños que Utilizan Pruebas Psicológicas: Áreas e Instrumentos Utilizados
}

\begin{abstract}
Resumen: El artículo tuvo como objetivo presentar una encuesta sobre el perfil de los psicólogos brasileños que utilizan pruebas psicológicas en lo que se refiere a su/s área/s de actuación, al uso de los instrumentos psicológicos en sus prácticas y a las pruebas que utilizan con mayor frecuencia en el ejercicio profesional. Participaron en el estudio 668 psicólogos, que ejercían la profesión en un promedio de 14,06 años $(\mathrm{DE}=11,34)$. Los datos fueron recopilados en la plataforma online por medio de un cuestionario que está siendo utilizado en varios países de América del Sur, divulgado a todos los psicólogos brasileños con apoyo de órganos profesionales. Los resultados obtenidos evidenciaron que la Psicología Clínica y de la Salud son las áreas más prevalentes de actuación principal de los psicólogos brasileños. Las pruebas psicológicas se utilizan principalmente para fines de psicodiagnóstico. Los grupos de edad más frecuentemente cubiertos por las evaluaciones realizadas son adultos. Las pruebas psicológicas más utilizadas se destinan a evaluar la personalidad o la inteligencia. La lista incluye Prueba Palográfica, HTP, Rorschach, Batería Factorial de Personalidad y las Escalas Wechsler de Inteligencia. Las pruebas proyectivas/expresivas o gráficas son las más usuales para evaluar la personalidad. La mayoría de las respuestas obtenidas en el análisis de las cinco pruebas más utilizadas se refieren a alguna prueba considerada favorable para su uso por Satepsi. Se destaca la extensión de la lista de las pruebas citadas, lo que indica que los psicólogos están atentos a los nuevos instrumentos y a las opciones de que disponen para calificar las evaluaciones realizadas. Sin embargo, hay un gran número de instrumentos utilizados que no tienen una evaluación certificada de sus propiedades técnicas.
\end{abstract}

Palabras clave: Evaluación Psicológica, Pruebas Psicológicas, Instrumentos Psicológicos.

\section{Introdução}

A avaliação psicológica permanece historicamente como prática identitária da profissão de psicólogo. Organismos internacionais representativos da Psicologia enquanto ciência e profissão defendem a realização exclusiva de avaliação psicológica por psicólogos, assumidos como os profissionais que mais entendem dos construtos psicológicos avaliados e dos instrumentos utilizados nessa avaliação.
Neste sentido, internacionalmente a avaliação psicológica permanece como uma das áreas importantes de investigação, tendo em vista assegurar aos investigadores e profissionais instrumentos atuais e válidos, bem como informações relevantes sobre a evolução nas atitudes e nos procedimentos de avaliação pelos psicólogos em sua prática (Archer, Buffington-Vollum, Stredny, \& Handel, 2006; Bornstei, 2016; Camara, Nathan, \& Puente, 2000; Wechsler et al., 2014). 
No Brasil, a profissão de psicólogo foi regulamentada em 1962. De acordo com a Lei no 4.119/1962 (Brasil, 1962), que segue vigente, as funções privativas do psicólogo no país referem-se à utilização de métodos e técnicas psicológicas com os seguintes objetivos: a) diagnóstico psicológico; b) orientação e seleção profissional; c) orientação psicopedagógica; e d) solução de problemas de ajustamento. Em todos esses casos, a avaliação psicológica pode estar envolvida como um recurso pertinente da prática profissional. No entanto, apesar de sua abrangência e relevância, a área da avaliação psicológica carece de estudos sobre seu estado da arte no Brasil, sobretudo no que tange ao perfil dos profissionais que utilizam testes psicológicos em suas práticas e aos testes mais usualmente utilizados nas avaliações (Reppold \& Noronha, 2018). Estudos internacionais já vêm sendo realizados nessa área, como os de Benson et al. (2019) e Evers et al. (2017).

Uma das poucas pesquisas de abrangência nacional investigando a formação e atuação dos psicólogos brasileiros foi realizada no final da década de 1980 por Bastos e Gomide (1989), em parceria com o Conselho Federal de Psicologia, com uma amostra de 2.448 psicólogos. Os resultados indicaram que, na ocasião, metade dos psicólogos formados não estava inscrita nos Conselhos Regionais de Psicologia e, portanto, não estava inserida no mercado de trabalho. Dentre os que exerciam a profissão, $60,7 \%$ atuavam em clínica, 23,6\% na área organizacional, $16,5 \%$ na escolar, $16,5 \%$ na docência, $5,6 \%$ na área comunitária, $3 \%$ na pesquisa e $5 \%$ em outras. No conjunto das atividades mais frequentemente desenvolvidas pelos psicólogos na época, a psicoterapia individual foi citada por $42,9 \%$ dos entrevistados, e a aplicação de testes foi a segunda mais frequente $(33,5 \%)$, sendo citada por psicólogos de todas as áreas de atuação e referida por $48 \%$ dos que trabalhavam na área organizacional. O psicodiagnóstico foi a terceira atividade mais frequentemente exercida por psicológicos, sendo mencionada por $28,1 \%$ dos entrevistados. Dentre os resultados, constatou-se também a hegemonia da Psicanálise como referencial teórico adotado, sendo a orientação mais citada em todas as áreas de atuação $(57,7 \%$ na Clínica, 30,9\% em Escolar e 22,0\% na Organizacional). A análise do comportamento e a fenomenologia apareceram a seguir, com, respectivamente, $8,7 \%$ e $6,8 \%$.

OConselho Federal de Psicologia (CFP) fez outros dois levantamentos sobre o tema em 2001 e 2004, os quais confirmaram a Clínica como área prevalente de atuação dos psicólogos brasileiros (Instituto Brasileiro de Opinião Pública e Estatística, 2004; World Health Organization, 2001). Os resultados de 2004 indicaram que $54,9 \%$ dos profissionais afirmavam que a Psicologia Clínica era sua principal área de atuação. A Psicologia da Saúde e a Psicologia Organizacional apareciam na sequência, sendo exercidas igualmente por $12 \%$ dos entrevistados na ocasião.

Investigando o panorama do trabalho dos psicólogos brasileiros, Bastos liderou estudo em 2010 que novamente constatou a primazia da Clínica como área de atuação, embora com tendência a diminuição (Bastos, Gondim, \& Borges-Andrade, 2010). Avaliadas as atividades exercidas em cada área, os autores concluíram que, apesar de constatarem que os campos de trabalho dos psicólogos foram ampliados de 1989 a 2010, as atividades desenvolvidas eram muito semelhantes, "inclusive aproximando os fazeres de diferentes áreas. Este é o caso, por exemplo, das atividades de avaliação psicológica, psicodiagnóstico e aplicação de testes" (p. 265), frequentemente citadas pelos entrevistados. Essa evidência reforça a importância da avaliação psicológica na formação profissional para diferentes áreas de atuação e indica que o uso de testes psicológicos deveria ser objeto de interesse da maior parte dos psicólogos brasileiros.

No entanto, a literatura cientifica indica que, no Brasil, a área da avaliação psicológica sofreu um período de desprestígio por parte dos psicólogos entre as décadas de 1960 e 2000. O início desse período fora marcado pela influência humanista e pela popularização da Psicanálise, com críticas aos pressupostos positivistas e suas técnicas. Algumas das principais queixas apontadas pelos profissionais no final do século XX eram relativas à qualidade dos instrumentos disponíveis (Hazboun \& Alchieri, 2013; Hutz, 2009; Hutz \& Bandeira, 2003; Noronha \& Reppold, 2010; Primi, 2010).

Noronha (2002) buscou identificar os problemas mais graves e mais frequentes no uso dos testes psicológicos, segundo a concepção de 214 psicólogos inscritos no CRP/6a Região, e listar os instrumentos mais utilizados. Os resultados obtidos indicaram que, na percepção dos psicólogos, os problemas mais graves eram alusivos aos instrumentos e seus usos - especificamente, problemas na construção, normatização e qualidade psicométrica dos instrumentos e no uso indevido, indiscriminado e mecânico dos testes, por pessoal não qualificado. Foram também citadas como problemas graves a supervalorização da análise quantitativa, a interpretação 
generalizada dos escores e o uso de um único instrumento como indicador definitivo de resultado da avaliação psicológica. Sobre o uso dos testes mais frequentes, 39,3\% dos participantes disseram não utilizar nenhum teste em sua atuação. Os dez instrumentos mais citados pelos respondentes foram: 1) WISC (15\%), 2) Teste de Apercepção Temática Infantil (13,9\%), 3) HTP (13,6\%), 4) Teste Gestáltico Viso-Motor Bender (13,2\%), 5) Teste de Apercepção Temática $(9,3 \%), 6)$ Teste de Wartegg $(9,3 \%), 7)$ Rorschach $(8,6 \%), 8)$ Teste de Desenhos e Histórias (6,8\%), 9) Teste de Matrizes Progressivas (Escalas Geral e Avançada) (6,4\%) e 10) Teste de Pirâmides Coloridas de Pfister $(3,9 \%)$. A partir dos dados, foi possível concluir que os tópicos de maior interesse para as avaliações na ocasião eram personalidade e inteligência e que a lista citada contemplava poucos construtos psicológicos. Esses resultados, de modo geral, vinham ao encontro do que era constatado em outros países por meio de grandes levantamentos internacionais, organizados por entidades como a American Psychological Association, a International Test Commission e a European Federation of Psychologists' Associations em diferentes décadas (Camara et al., 2000; Evers et al., 2012, 2017; Lubin, Larsen, \& Matarazzo, 1984; Muñiz, Prieto, Almeida, \& Bartram, 1999; Oakland, Douglas, \& Kane, 2016; Sundberg, 1961).

Em 2005, Noronha, Primi e Alchieri investigaram quais eram os instrumentos mais conhecidos/utilizados de estudantes e profissionais de Psicologia do Brasil. Para tanto, constituíram uma amostra de 304 participantes, oriundos de 18 estados brasileiros das cinco regiões do país. Os dados foram coletados em um evento científico nacional, por meio de um formulário que listava os 145 instrumentos comercializados no Brasil na ocasião. Os resultados indicaram que o número de instrumentos desconhecidos/não utilizados era maior do que o de conhecidos/utilizados e que as técnicas mais conhecidas/utilizadas eram, como no estudo de Noronha (2002), referentes à avaliação da personalidade. Dentre os mais assinalados, encontravam-se o Rorschach, o Wartegg e o Zulliger na avaliação da personalidade, e o G-36 e o WISC na avaliação da inteligência. Nesse artigo, os pesquisadores concluíram que os resultados indicavam um "cenário lastimável” (p. 398), pois pouquíssimos dos instrumentos disponíveis eram conhecidos dos participantes, fato que remetia, na concepção dos autores, ao equívoco dos psicólogos brasileiros de não tornar prática de sua formação continuada a leitura sistemática de periódicos científicos. Essas dificuldades eram, na época, também observadas em outros países da América Latina (Muñiz et al., 1999), que tinham forte dependência de instrumentos estrangeiros, utilizados, muitas vezes, sem adequados estudos de adaptação.

Diante dessa realidade e de uma demanda social, o Conselho Federal de Psicologia criou, em 2003, o Sistema de Avaliação dos Testes Psicológicos (Satepsi) com o propósito de estabelecer critérios mínimos de qualidade dos testes psicológicos disponíveis para uso profissional, indicar quais testes são de uso favorável ou desfavorável, de acordo com esses critérios, e divulgar informações sobre a área de avaliação psicológica no Brasil, como estabelece a Resolução no 2 de 2003 do CFP (2003). Os resultados ao longo dos últimos anos indicam que o sistema tem sido bem-sucedido em qualificar os recursos da área (Noronha, \& Reppold, 2010; Primi, 2010; Reppold \& Noronha, 2018; Wechsler et al., 2014), tornando-se uma referência internacional na certificação de instrumentos psicológicos.

No entanto a pesquisa realizada por Hazbourn e Alchieri (2013) indica que ainda é alto o número de profissionais que questionam ou rejeitam o uso da avaliação psicológica em suas práticas e mantêm uma visão equivocada sobre o uso de testes psicológicos. Para investigar as concepções de psicólogos sobre o uso de testes, o estudo constituiu uma amostra de 932 participantes. Destes, 278 afirmaram não realizar avaliação psicológica em suas práticas. Questionados sobre as razões para o não uso de avaliação psicológica em sua práxis, os respondentes alegaram ser em prol 1) da natureza do trabalho que exerciam, 2) do foco do trabalho na atuação clínica, 3) do uso de outro processo avaliativo que não um teste psicológico, 4) da crença de que a avaliação psicológica não faz parte das atividades de sua área ou de sua abordagem e 5) da precariedade de sua formação acadêmica. Quanto à natureza do trabalho, chama atenção a concepção errônea/obsoleta de avaliação psicológica como sinônimo do uso de testes. Cite-se que a maioria dos psicólogos que informaram crer que as práticas de avaliação psicológica eram desnecessárias ou incompatíveis com sua área de atuação exercia atividades no campo da assistência social ou da psicoterapia individual/familiar, o que remete a uma reflexão sobre a formação dos psicólogos brasileiros na área da avaliação psicológica e sobre as dificuldades que essa concepção equivocada implica para a avaliação 
da efetividade de práticas exclusivamente intervencionistas exercidas pelos psicólogos nesses campos.

$\mathrm{O}$ interesse da classe em conhecer a opinião dos psicólogos sobre o uso de testes e as práticas da área da avaliação é internacional. Um pesquisa realizada com psicólogos espanhóis (Muñiz \& FérnandezHermida, 2010) por meio de um questionário desenvolvido pela Comisión de Tests de la Federación Europea de Asociaciones de Psicólogos, por exemplo, indicou que os espanhóis demonstravam atitudes positivas frente ao uso dos testes, ainda que estivessem bastante cautelosos quanto ao uso de testes computadorizados e preocupados em oferecer melhor formação na área da avaliação psicológica e da psicometria. A discussão desse estudo apresenta comparações dos achados com o perfil da área em outros países do contexto europeu e norte-americano, mas uma das considerações que os autores fazem é referente à falta de estudos nessa linha em países ibero-americanos, o que permitiria uma comparação transcultural. Os autores reconhecem que estudar as práticas de avaliação psicológica, as técnicas usadas e as próprias preocupações dos psicólogos nesta área ilustra a evolução da psicologia como ciência e profissão em cada país.

Então, diante da necessidade de conhecer melhor o perfil do psicólogo que utiliza testes em países ibero-americanos, decidiu-se replicar o estudo espanhol. Assim sendo, com o apoio do Colégio de Psicólogos da Espanha e do Instituto Brasileiro de Avaliação Psicológica, foi iniciado um levantamento on-line, visando a investigar as atitudes e as práticas de psicólogos da área de avaliação psicológica. Especificamente, o presente artigo apresenta um levantamento sobre o perfil dos psicólogos brasileiros que utilizam testes psicológicos no que tange a sua(s) área(s) de atuação, ao uso que fazem dos instrumentos psicológicos em suas práticas e aos testes que utilizam com maior frequência no exercício profissional.

\section{Método}

\section{Amostra}

Participaram do estudo 668 psicólogos, sendo $81,9 \%$ do sexo feminino, com média de idade de 40,4 anos $(D P=12,67)$. Quanto à formação dos respondentes, $45,0 \%$ tinham título de especialista, 20,6\% eram mestres e 21,4\% eram doutores. A Psicologia Clínica e da Saúde surge como a área mais citada dentre aquelas nas quais os cursos de pós-graduação referidos se inseriam. O número de anos de prática como psicólogos oscilou entre 1 e 48 anos, situando-se a média em 14,1 anos $(D P=11,34)$. A amostra contou com participantes das cinco regiões demográficas do Brasil.

\section{Instrumento}

O instrumento utilizado no levantamento foi um questionário eletrônico empregado em estudo espanhol (Muñiz \& Férnandez-Hermida, 2010), traduzido e adaptado para o português brasileiro, o qual era precedido por Termo De Compromisso Livre e Esclarecido referente à participação no estudo. O questionário era composto por 33 questões (caracterizadas como perguntas fechadas ou questões abertas) sobre a formação da(o) psicóloga(o), áreas de atuação, testes mais utilizados, dificuldades e sugestões para melhorar a área de avaliação psicológica no Brasil.

\section{Procedimentos de coleta e análise de dados}

Por meio de divulgação do Conselho Federal de Psicologia, do Instituto Brasileiro de Avaliação Psicológica e da Associação Nacional de Pesquisa e PósGraduação em Psicologia, todos os psicólogos inscritos nessas entidades receberam informação sobre o estudo e o convite para dele participarem de forma voluntária. Os dados foram coletados de forma eletrônica em uma plataforma on-line de coleta de dados. O preenchimento do questionário demorava em torno de 10 minutos. A plataforma permaneceu aberta para preenchimento no período de maio de 2017 a janeiro de 2018. Os dados qualitativos do instrumento foram analisados por meio de análises descritivas (análise de conteúdo), e os dados quantitativos por meio de análises descritivas (análise de frequência/percentual, média e desvio-padrão).

O levantamento faz parte de um estudo internacional envolvendo diversos países. Seu planejamento levou em consideração o cumprimento das orientações internacionais em matéria de exigências éticas para pesquisa com seres humanos adultos, sendo assegurados aos respondentes a confidencialidade e o anonimato das informações, bem como o caráter voluntário de sua participação, expresso por meio de consentimento livre e informado.

\section{Resultados}

A Tabela 1 mostra a área de atuação principal declarada pelos psicólogos entrevistados. Apresenta 
também o percentual de psicólogos que exercem alguma atividade profissional na área citada, considerando que respondentes poderiam indicar atividades em mais de uma área de atuação. Mais da metade dos participantes informou exercer atividade em duas ou mais áreas de atuação profissional (50,14\%).

\section{Tabela 1}

Distribuição da amostra conforme área de atuação autodeclarada como principal e conforme área na qual o participante exerce alguma atividade profissional $(\mathrm{n}=622)$.

\begin{tabular}{lcc}
\hline \multicolumn{1}{c}{$\begin{array}{c}\text { Área de atuação } \\
\text { profissional }\end{array}$} & $\begin{array}{c}\text { Atuação } \\
\text { principal } \\
\text { (\%) }\end{array}$ & $\begin{array}{c}\text { Alguma } \\
\text { atividade } \\
\text { na área (\%) }\end{array}$ \\
\hline $\begin{array}{l}\text { Psicologia Clínica e da } \\
\text { Saúde }\end{array}$ & 33,92 & 67,84 \\
$\begin{array}{l}\text { Ensino Superior e } \\
\text { Investigação }\end{array}$ & 15,59 & 31,83 \\
$\begin{array}{l}\text { Neuropsicologia } \\
\text { Psicologia do Trabalho } \\
\text { e das Organizações }\end{array}$ & 11,58 & 29,90 \\
$\begin{array}{l}\text { Psicologia Escolar e da } \\
\text { Educação }\end{array}$ & 11,41 & 24,11 \\
$\begin{array}{l}\text { Psicologia Forense e da } \\
\text { Justiça }\end{array}$ & 4,85 & 20,25 \\
$\begin{array}{l}\text { Psicologia Social e } \\
\text { Comunitária }\end{array}$ & 3,86 & 11,89 \\
$\begin{array}{l}\text { Psicologia da Atividade } \\
\text { Física e do Desporto }\end{array}$ & 0,32 & 1,44 \\
Outra & 12,55 & 20,25 \\
\hline
\end{tabular}

Questionados se, durante o exercício profissional, faziam uso de testes psicológicos habitualmente, 600 participantes responderam à questão, e 94,33\% afirmaram que sim $(n=566)$. Dentre as razões apontadas, em uma lista de múltipla escolha, como justificativa para o não uso dos testes, os respondentes alegaram falta de recursos para compra de instrumentos (50\%), falta de formação adequada para uso de testes (35,29\%), falta de confiança nos testes como recurso para a prática profissional $(11,76 \%)$ e outros $(50 \%)$. A falta de estudos de validação dos testes brasileiros foi assinalada por apenas um participante.

Dentre os que afirmavam utilizar testes, a média do percentual de uso associado a cada atividade foi: $44,22 \%$ para fins psicodiagnósticos, $15,11 \%$ para investigação científica, 10,64\% para recrutamento e seleção de pessoal, $10,1 \%$ para docência, 8,09\% para orientação ou aconselhamento, $8,03 \%$ para fins de triagem e $6,6 \%$ para avaliação de programas e intervenções. De acordo com os resultados, os testes utilizados pelos participantes destinam-se a avaliar principalmente adultos entre $26 \mathrm{e}$ 64 anos de idade (média de uso $=30,32 \%$ ) e indivíduos entre 13 e 25 anos de idade (média de uso $=25,24 \%$ ). O percentual médio do uso dos testes entre crianças de 8 a 12 anos foi $15,75 \%$; entre crianças de 3 a 7 anos, foi $12,72 \%$; entre crianças de 0 a 3 anos, $2,03 \%$; e entre adultos com idade superior a 65 anos, $6,36 \%$.

Sobre os testes mais utilizados pelos psicólogos, a questão foi respondida por apenas 440 respondentes. A Tabela 2 apresenta as respostas mais frequentemente citadas pelos participantes como primeira opção à questão "Quais testes você utiliza com mais frequência no exercício de sua profissão? Cite o nome dos testes, ordenando-os de acordo com a frequência de uso". A análise dos dados referentes à primeira opção de resposta dos participantes revela 99 respostas diferentes, sendo 60 testes considerados favoráveis para uso profissional segundo avaliação do Satepsi, 3 testes desfavoráveis pelo Satepsi (Fábulas de Düss, Bateria de Testes de Atenção e Bateria de Rotas de Atenção), 26 instrumentos não avaliados pelo Satepsi e 10 classes de respostas referentes a construtos psicológicos (por exemplo, inteligência, personalidade, atenção) ou tipo de instrumento (como escalas, inventários, testes projetivos).

\section{Tabela 2}

Lista das respostas mais frequentes à questão "Quais testes você utiliza com mais frequência no exercício de sua profissão" $(\mathrm{n}=440)$.

\begin{tabular}{cccc}
\hline Resposta & $\begin{array}{c}\text { Construto } \\
\text { avaliado }\end{array}$ & N & $\%$ \\
\hline Palográfico & Personalidade & 69 & 15,68 \\
WISC & Inteligência & 49 & 11,13 \\
HTP & Personalidade & 43 & 9,77 \\
Rorschach & Personalidade & 32 & 7,27 \\
Bateria Fatorial & Personalidade & 21 & 4,77 \\
de Personalidade & & & \\
Escalas Wechsler & Inteligência & 17 & 3,86 \\
de Inteligência & Inteligência & 16 & 3,63 \\
WAIS & - & 12 & 2,72 \\
Inteligência & Ansiedade, & & \\
& depressão, & 10 & 2,27 \\
Escalas Beck & desesperança e & & \\
& ideação suicida & & 2,04 \\
Personalidade & - & 9 & 2,04 \\
Pfister & Personalidade & 9 & \\
\hline
\end{tabular}


Considerando que o participante poderia emitir múltiplas respostas para a questão "Quais testes você utiliza com mais frequência no exercício de sua profissão", realizou-se uma análise de frequência das cinco primeiras respostas apresentadas pelo respondente. Os resultados obtidos somaram 1.880 respostas, sendo 1.601 relativas a algum teste psicológico aprovado pelo Satepsi. A análise indicou 248 respostas diferentes: 97 testes considerados favoráveis para uso profissional segundo avaliação do Satepsi, 9 testes desfavoráveis pelo Satepsi (Bateria de Rotas de Atenção, Bateria de Testes de Atenção, Fábulas de Duss, Inventário de Ansiedade Traço Estado (Idate), Inventário Fatorial de Personalidade (IFP), Medida de Fluência Verbal, QSG, Testes de Atenção
Discriminativa e Teste das Figuras - Inteligência Geral Não Verbal), 105 instrumentos não avaliados pelo Satepsi e respostas classificadas em 25 categorias referentes a construtos psicológicos (por exemplo, motivação, função executiva, impulsividade, memória, ansiedade, desenvolvimento etc.) ou tipo/finalidade do instrumento (como escalas Likert, testes neuropsicológicos, testes projetivos, inventários, escalas de contrato psicológico, testes profissiográficos).

A Tabela 3 apresenta as respostas mais frequentemente citadas pelos participantes considerando o agrupamento das cinco primeiras opções à questão "Quais testes você utiliza com mais frequência no exercício de sua profissão? Cite o nome dos testes, ordenando-os de acordo com a frequência de uso".

\section{Tabela 3}

Lista dos testes mais frequentemente utilizados pelos psicólogos, considerando o agrupamento das cinco primeiras respostas à questão "Quais testes você utiliza com mais frequência no exercício de sua profissão".

\begin{tabular}{|c|c|c|c|c|c|}
\hline Teste & Construto avaliado & Indicação de uso & $\mathrm{N}$ & $\% *$ & $\% * *$ \\
\hline WISC & Inteligência & 6 anos a 16 anos e 11 meses & 205 & 10,90 & 12,80 \\
\hline HTP & Personalidade & Acima de 8 anos & 147 & 7,81 & 9,18 \\
\hline Palográfico & Personalidade & Adolescentes e adultos & 141 & 7,50 & 8,80 \\
\hline $\mathrm{R} 1$ & Inteligência não verbal & Adultos & 79 & 4,20 & 4,93 \\
\hline Teste AC & Atenção concentrada & Adolescentes e adultos & 79 & 4,20 & 4,93 \\
\hline BFP & Personalidade & Adolescentes e adultos & 77 & 4,09 & 4,80 \\
\hline Rorschach & Personalidade & Adultos ${ }^{* * * *}$ & 71 & 3,77 & 4,43 \\
\hline TAT & Personalidade & 14 a 40 anos & 67 & 3,56 & 4,18 \\
\hline WAIS & Inteligência & 16 a 89 anos & 66 & 3,51 & 4,12 \\
\hline Pfister & Personalidade & $\begin{array}{l}\text { Versão Infantil: } 6 \text { a } 14 \text { anos; } \\
\text { Versão Adulta: } 18 \text { a } 66 \text { anos. }\end{array}$ & 59 & 3,13 & 3,68 \\
\hline BPA & Atenção & 6 a 82 anos & 45 & 2,39 & 2,81 \\
\hline Escalas Beck & $\begin{array}{l}\text { Ansiedade, depressão, } \\
\text { desesperança, ideação suicida }\end{array}$ & 17 a 80 anos & 33 & 1,75 & 2,06 \\
\hline CAT & Personalidade & $\begin{array}{l}\text { CAT-A: } 5 \text { a } 10 \text { anos; CAT-H: } 7 \text { a } 12 \\
\quad \text { anos e } 11 \text { meses }\end{array}$ & 29 & 1,54 & 1,81 \\
\hline $\begin{array}{l}\text { Escalas Wechsler } \\
\text { de Inteligência*** }\end{array}$ & Inteligência & - & 28 & 1,48 & 1,74 \\
\hline Zulliger & Personalidade & Adultos $* * * *$ & 28 & 1,48 & 1,74 \\
\hline \multicolumn{6}{|c|}{ *A coluna indica o percentual de citações levando em conta o total das respostas consideradas ( $\mathrm{n}=1.880$ ). } \\
\hline \multicolumn{6}{|c|}{$\begin{array}{l}\text { **A coluna indica o percentual de citações levando em conta as respostas que citavam um teste psicológico aprovado pelo } \\
\text { Satepsi }(\mathrm{n}=1.601) \text {. }\end{array}$} \\
\hline \multicolumn{6}{|c|}{$\begin{array}{l}\text { *** Embora o WISC e o WAIS sejam exemplos de Escalas Wechsler de Inteligência, as respostas foram mantidas separadas para } \\
\text { garantir a fidelidade dos dados. }\end{array}$} \\
\hline \multicolumn{6}{|c|}{ **** Dependendo da versão do teste, o instrumento é indicado para uso a partir dos 16 anos de idade. } \\
\hline \multicolumn{6}{|c|}{$\begin{array}{l}\text { Abreviaturas - WISC: Escalas Wechsler de Inteligência para Crianças; HTP: Teste Casa-Árvore-Pessoa; Teste AC: Teste de } \\
\text { Atenção Concentrada; BFP: Bateria Fatorial de Personalidade; TAT: Teste de Apercepcão Temática; WAIS: Escalas Wechsler de } \\
\text { Inteligência para Adultos; BPA: Bateria Psicológica para Avaliação da Atenção; CAT: Teste de Apercepção Temática (CAT-A: } \\
\text { Forma Animal; CAT-H: Forma Humana). }\end{array}$} \\
\hline
\end{tabular}




\section{Discussão}

O presente estudo teve como objetivo apresentar um levantamento sobre o perfil dos psicólogos brasileiros que utilizam testes psicológicos no que tange a sua(s) área(s) de atuação, ao uso que fazem dos instrumentos psicológicos em suas práticas e aos testes que utilizam com maior frequência no exercício profissional. Os resultados obtidos evidenciaram que a Psicologia Clínica e da Saúde são as áreas mais prevalentes de atuação principal dos psicólogos brasileiros, tal qual fora constatado nos levantamentos anteriores realizados em 1989 (Bastos \& Gomide, 1989), 2001 (World Health Organization, 2001), 2004 (Instituto Brasileiro de Opinião Pública e Estatística, 2004) e 2010 (Bastos et al., 2010). Em 2004, 54,9\% dos profissionais afirmavam que a Psicologia Clínica era a sua principal área de atuação. Na atualidade, mais de dois terços dos psicólogos $(67,84 \%)$ informaram realizar alguma atividade nas áreas da Clínica e da Saúde. Em comparação com levantamentos anteriores, destaca-se a alta frequência com que os participantes estão atualmente envolvidos em atividades do ensino superior e investigação (31,83\%). Isso pode ter ocorrido devido à divulgação do questionário por parte de associações científicas, atingindo um número maior de pesquisadores.

Ressalta-se a diversidade das atividades desenvolvidas pelos psicólogos: aproximadamente um terço da amostra exerce atividades na área da Neuropsicologia, um quarto na área da Psicologia Organizacional e do Trabalho e um quinto na área da Psicologia Escolar e Educacional. Esses resultados vão ao encontro da proposta de uma formação generalista que é característica das Diretrizes Curriculares dos Cursos de Graduação em Psicologia no Brasil, instituídas pela Resolução do Ministério da Educação no 5/2011 (Brasil, 2011). Todavia, como já apontado por Bastos et al. (2010), levam à reflexão sobre os limites tênues que demarcam as especificidades de algumas áreas em termos práticos e sobre o real domínio dos profissionais em relação a técnicas e intervenções próprias de diferentes áreas de exercício profissional.

No entanto, essa não é uma discussão apenas de âmbito nacional. Publicações estrangeiras também indicam que a formação em países da Europa, da América do Norte e da América Latina é generalista e que os profissionais tendem a desenvolver atividades simultâneas em diferentes áreas (Evers et al., 2012, 2017; Muñiz \& Férnandez-Hermida, 2010; Muñiz et al., 1999; Wechsler et al., 2014). Em geral, aqueles envolvidos na área da Neuropsicologia são os que mais frequentemente realizam atividades envolvendo avaliação psicológica. Em um levantamento estadunidense realizado por Camara et al. (2000), do qual participaram 1.002 membros da Associação Nacional de Neuropsicologia (NAN) e 1.500 psicólogos vinculados à American Psychological Association (APA), aleatoriamente selecionados, constatou-se que a maioria dos neuropsicólogos dedica parte substancial do tempo de suas práticas profissionais a avaliação: $54 \%$ dos neuropsicológicos informaram habitualmente usar mais de 15 horas semanais para administração, correção ou interpretação de testes psicológicos.

Também na Europa, uma avaliação positiva sobre o uso de testes psicológicos tem sido descrita nas pesquisas coordenadas pela Comisión de Tests de la Federación Europea de Asociaciones de Psicólogos (Muñiz \& Férnandez-Hermida, 2010), como a que deu origem ao presente estudo, ou nos estudos desenvolvidos por Evers et al. $(2012,2017)$ a partir da investigação em 29 países, sendo crescente o relato dos profissionais que afirmam utilizar testes de forma habitual em suas práticas em todos os países. Nesta pesquisa, os resultados corroboram essa tendência, e indicam que o uso de testes psicológicos durante as práticas profissionais é frequente, principalmente quando se trata da realização de psicodiagnóstico.

Chama atenção o alto percentual de respondentes que afirmaram utilizar testes psicológicos em suas práticas $(94,33 \%)$. Esse dado parece refletir a confiança dos psicólogos nos avanços nacionais da área nos últimos anos e configura um panorama sobre a avaliação psicológica brasileira diferente daquele que era observado na década de 1990. A qualificação da área no Brasil é evidente também em outros estudos internacionais recentemente publicados. Um deles (Evers et al., 2017), uma pesquisa mundial desenvolvida em conjunto pela International Test Commission e pela European Federation of Psychologists' Associations, investigou a opinião dos 20.467 psicólogos sobre práticas de avaliação psicológica. Essa pesquisa incluiu profissionais de 29 países, inclusive o Brasil, que responderam a cinco escalas avaliativas. Os tópicos abordados nas escalas foram: E1) preocupação sobre o uso incorreto do teste, E2) regulamentação de testes psicológicos, E3) uso de testes informatizados, E4) avaliação sobre os testes disponíveis e E5) formação e conhecimento relacionados ao uso de testes. Os resultados obtidos indicam que os 
psicólogos brasileiros valorizam a área da avaliação psicológica e os recursos disponíveis. O índice de preocupação dos brasileiros em relação ao uso de testes não difere do que, em geral, é observado nos demais países. Os tamanhos de efeito obtidos na comparação entre os brasileiros e os demais participantes foram: E1) $d=-0,02$; E2) $d=0,94$; E3) $d=0,21$; E4) $d=1,24$. Os deltas nas Escalas 2 e 4 indicam, respectivamente, que os brasileiros têm uma visão mais favorável à regulamentação dos testes psicológicos e maior crença de que os testes psicológicos são valorosos à Psicologia e a sua prática profissional especificamente. O escore médio dos brasileiros na escala 4 foi o maior entre os 29 países ( $M=4,66 ; D P=0,46$; intervalo entre 1 e 5), o que é um dado surpreendentemente positivo.

No entanto, nesta pesquisa, o dado que indica que 94,33\% dos psicólogos utilizam testes psicológicos em suas práticas deve ser visto com cautela, pois é possível que esse dado esteja superdimensionado por um viés de recrutamento dos participantes do estudo, visto que sua divulgação informava que o levantamento era referente ao uso de testes psicológicos. Assim, provavelmente era de interesse específico daqueles envolvidos com avaliação psicológica. Esse viés de recrutamento pode também explicar, em parte, a baixa adesão dos psicólogos ao estudo, visto que a divulgação supostamente alcançou todos os psicólogos vinculados ao Conselho Federal de Psicologia (CFP) - 309.012 profissionais (Conselho Federal de Psicologia, 2018) -, mas apenas 668 participaram do levantamento.

Os dados obtidos juntos àqueles que informaram não utilizar testes psicológicos em suas práticas reforçam os argumentos da falha da formação acadêmica e do alto custo dos instrumentos, já expostos em outros estudos (Noronha, 2002, Noronha \& Reppold, 2010; Hazbourn \& Alchieri, 2013). Porém apresentam uma nova perspectiva: enquanto nos estudos prévios, a principal razão alegada pelos psicólogos para não uso de testes era referente à baixa qualidade técnica dos instrumentos disponíveis, no presente levantamento apenas um participante assinalou a opção relativa à falta de estudos de validação dos testes brasileiros como motivo para não uso de testes em seu exercício profissional. Provavelmente esse dado seja um reflexo positivo do esforço do CFP, das entidades científicas, das editoras e dos pesquisadores em dignificar a área da avaliação psicológica brasileira nos últimos anos, seja estabelecendo critérios mínimos de qualidade dos testes psicológicos, no caso do Satepsi, seja fomentando novas pesquisas, disponibilizando material qualificado ou promovendo o debate na área.

Coerente com o fato de que a Psicologia Clínica e da Saúde são as áreas mais frequentes de exercício profissional dos respondentes, os resultados obtidos indicam que os psicólogos brasileiros fazem uso de testes psicológicos principalmente para fins de psicodiagnóstico (44,22\%). Revelam também que os grupos etários mais frequentemente cobertos pelas avaliações realizadas são adultos entre 26 e 64 anos (média de uso $=30,32 \%$ ) e indivíduos entre 13 e 25 anos de idade (média de uso $=25,24 \%$ ). Uma vez que a maioria dos testes considerados favoráveis para uso profissional atualmente no país é destinada a adultos e adolescentes (Reppold, Serafini, Gurgel, \& Kaiser, 2017; Reppold, Serafini, Ramires, \& Gurgel, 2017), esse dado indica, por um lado, haver uma correspondência entre as demandas da área e a disponibilidade do mercado. Por outro, pode denotar que o uso de testes para avaliação de crianças é menos frequente devido à menor oferta de instrumentos qualificados e/ou ao maior uso de recursos de observação ou entrevistas sobre conduta ou desenvolvimento como fontes primárias de informação.

Quanto aos testes mais frequentemente utilizados pelos psicólogos brasileiros, os resultados evidenciam que, tal como ocorre em estudos internacionais (Evers et al., 2012, 2017; Muñiz \& Férnandez-Hermida, 2010; Wechsler et al., 2014), a maior demanda é por testes que avaliam os construtos personalidade e inteligência. Destaque também à avaliação da atenção. A lista dos instrumentos citados como de uso de mais frequente no presente levantamento é semelhante à encontrada em estudos prévios nacionais (Noronha, 2002; Noronha et al., 2005) e estrangeiros, sejam esses recentes (Evers et al., 2012, 2017; Wechsler et al., 2014) ou mais antigos (Lubin et al., 1984; Sundberg, 1961). Dos dez instrumentos descritos por Noronha em 2002 como os mais utilizados na prática dos psicólogos brasileiros, seis se repetem aqui, na lista apresentada na Tabela 3 (WISC, TAT, HTP, CAT, Rorschach e PFISTER). Cite-se que dois dos testes listados por Noronha (2002) atualmente não são mais considerados favoráveis para uso em exercício profissional no Brasil (Wartegg e Teste de Desenho e História). Em ambos os estudos, os testes mais frequentemente listados para avaliação do construto personalidade são testes projetivos, expressivos ou gráficos. 
Nesta pesquisa e nos demais levantamentos nacionais sobre os testes mais utilizados pelos psicólogos aqui referenciados, os resultados obtidos se aproximam dos levantamentos internacionais em dois aspectos: estabilidade temporal das listas dos "dez instrumentos mais utilizados" e predomínio de testes adaptados à cultura local. No estudo espanhol de Muniz e Férnandez-Hermida (2010), por exemplo, os instrumentos mais utilizados pelos psicólogos foram o WISC (Wechsler Intelligence Scale for Children), o 16 PF (16 Personality Factors) e o MCMI (Millon Clinical Multiaxial Inventory). Também nos estudos desenvolvidos pela International Test Commission e pela European Federation of Psychologists' Associations observa-se que o padrão conservador dos psicólogos na escolha dos instrumentos tem se repetido ao longo dos anos, em diferentes países, dentre os quais Áustria, Bélgica, Alemanha, República Tcheca, Croácia, Lituânia, Noruega, Polônia, Romênia, Turquia, Eslováquia, Espanha e Reino Unido (Evers et al., 2012). Os testes comumente utilizados nesses países são instrumentos clássicos de inteligência e personalidade, como WISC (26,9\%), WAIS (19,3\%), MMPI $(12,7 \%)$, Raven (12\%) e 16 PF (8,8\%). OWISC consta na lista dos dez instrumentos mais frequentes em 16 dos 17 países europeus investigados; o WAIS, em 14, e o MMPI e o RAVEN, em 10. Nesses países, há clara predominância de testes psicométricos em relação aos projetivos, sendo o Rorschach o único teste projetivo na lista geral dos instrumentos de uso mais frequentes apresentada por Evers e colaboradores (2012). No entanto, variações entre os países são observadas. Em seis países, nenhum teste projetivo consta na lista dos dez mais frequentemente utilizados (Croácia, Alemanha, Holanda, Noruega, Suécia e Reino Unido). Na República Tcheca e na Romênia, três testes projetivos estão incluídos na lista; nos demais países investigados, dois (Ever et al., 2012). O predomínio dos instrumentos psicométricos na Europa contrasta com o uso frequente das técnicas projetivas nos países latino-americanos, constatada por Muñiz et al. (1999) e por Wechsler et al. (2014), e confirmada nos dados apresentados por Noronha (2002) e neste estudo.

O padrão conservador da escolha de testes clássicos é observado também na pesquisa organizada por Oakland, Douglas e Kane (2016). Nesse estudo, os autores investigaram quais eram os dez testes padronizados mais frequentemente utilizados para avaliação de crianças e adolescentes por psicólogos escolares de 64 países. No ranking geral, os testes listados foram WISC (mencionado por $80 \%$ da amostra), Matrizes Progressivas de Raven (40\%), WPPSI (Wechsler Preschool and Primary Scale of Intelligence- 27\%), CBCL (Child Behavior Checklist/Achenbach- 27\%), Teste Gestáltico Bender (25\%), CAT/TAT (19\%), KABC (Kaufman Assessment Battery for Children - 17\%), Desenho da Pessoa/Figura Humana (16\%), StanfordBinet (13\%) e WAIS (Wechsler Adult Intelligence Scale$13 \%)$. Os resultados indicaram que nove dos testes listados eram referenciados em normas (percentis), e oito eram destinados especificamente a crianças e adolescentes. Oito dos testes avaliavam habilidades cognitivas, sendo sete específicos para inteligência. Os dois demais avaliavam personalidade ou comportamento. Todos, com exceção das Matrizes de Raven, eram testes administrados individualmente.

A pesquisa buscava atualizar resultados observados 24 anos antes. Em comparação aos resultados obtidos em 1989, os dados coletados em 2013 indicaram que o WISC e o Matrizes Progressivas de Raven permaneceram no topo da lista de preferência nos 64 países e que o Bender, o Stanford-Binet e o WAIS se mantiveram entre os escolhidos. Segundo os autores, esta estabilidade se deve a diferentes fatores. Entre eles, o fato de os psicólogos se sentirem confortáveis com o uso de um instrumento de que tenham domínio, as boas propriedades psicométricas apresentadas por esses instrumentos, as questões financeiras que limitam a compra de novos testes e a falta de regulamentação dos testes em muitos países, que não impede o uso de versões antigas dos testes disponíveis. Cite-se que as três primeiras razões podem explicar também, na presente pesquisa, a estabilidade da lista dos testes mais utilizados pelos psicólogos brasileiros, quando comparados os resultados atuais com os dados publicados por Noronha (2002).

Outro dado interessante descrito por Oakland e colaboradores (2016) é uma comparação entre a lista dos países menos desenvolvidos $(n=22)$, emergentes ( $n=24)$ e avançados $(n=18)$, a qual indica resultados semelhantes entre todos os países. O WISC e as Matrizes Progressivas de Raven se mantiveram no topo da preferência nas três categorias para avaliação infanto-juvenil. Nos países emergentes, o Bender e o DFH foram mais prevalentes do que em outros locais, o que foi justificado pelos autores em razão de serem instrumentos de lápis-e-papel, economicamente mais acessíveis. Já nos países 
subdesenvolvidos, constatou-se que o uso da WAIS, escala originalmente destinada para adultos, é muito mais frequente do que nos demais países, o que chama atenção, visto que a maioria das crianças avaliadas tem idade entre 6 e 12 anos. Em todos os casos, é fato que a maioria dos testes listados nos 64 países são instrumentos americanos ou franceses, que requerem estudos de adaptação e de busca de evidências de validade no contexto local, o que em alguns locais implica dificuldades consideráveis.

\section{Considerações finais}

Os dados apresentados no presente levantamento indicam que testes considerados desfavoráveis pelo Satepsi continuam sendo utilizados por alguns psicólogos brasileiros, sendo alguns desses instrumentos classificados como desfavoráveis pelo Conselho desde a década passada. Nessa linha, merece reflexão o fato de que a maioria dos instrumentos listados pelos participantes $(n=105)$ não consta na lista dos instrumentos avaliados pelo Satepsi. Ressalta-se que a questão formulada era explícita ao relacionar o uso do teste ao exercício profissional e que somente $31,83 \%$ dos participantes afirmavam exercer atividade de docência ou investigação científica, o que poderia justificar o uso de instrumentos desfavoráveis em atividades de pesquisa. Também se observa com preocupação o fato de que parte dos psicólogos não soube/ não quis mencionar o nome dos testes que utilizam, limitando-se a nomear os construtos que avaliam ou o tipo e/ou finalidade dos instrumentos que utilizam (mencionam, por exemplo, inventários, escalas Likert, escalas neuropsicológicas ou escalas de contrato terapêutico). Em contrapartida, constata-se que a maioria das respostas obtidas na análise dos cinco testes mais utilizados refere-se a alguns testes que apresentam requisitos mínimos de qualidade, de novo considerando os critérios adotados pela avaliação do Satepsi. Das 1.880 respostas, 1.601 listavam um teste favorável, sendo que 97 testes psicológicos da lista dos testes aprovados pelo Satepsi foram mencionados. Visto que atualmente a lista do Satepsi é formada por cerca de 180 testes favoráveis e que muitos dos testes citados são publicações recentes, a heterogeneidade das respostas sugere que os psicólogos estão atentos aos novos instrumentos e às opções de que dispõem para qualificar as avaliações realizadas. Uma das limitações do estudo é o fato de o questionário utilizado não incluir questões específicas sobre o Satepsi, por se tratar de um levantamento planejado em âmbito internacional, incluindo o mesmo instrumento traduzido. Outra limitação é o alcance da amostra constituída. Embora o levantamento tenha sido divulgado aos psicólogos pelo Conselho Federal de Psicologia e por outras entidades científicas a todos os psicólogos brasileiros, o número de respondentes foi 668 .

De qualquer forma, a situação descrita a propósito dos testes utilizados merece reflexão e novos estudos. Por um lado, é possível que, no contexto acadêmico, os docentes escolham ensinar o uso de instrumentos que não se encontrem em situação favorável para uso profissional, segundo avaliação do Satepsi. Esta situação não necessariamente entra em conflito com as preocupações éticas e técnicas que determinam que os testes a serem utilizados pelos psicólogos como fonte primária de informação devem apresentar evidências de validade e normas, pois não estamos face a sua utilização no exercício profissional da avaliação psicológica. Motivos vários podem estar na origem dessa utilização nas aulas; por exemplo, a perspectiva histórica da avaliação psicológica de certos construtos ou de certas problemáticas. É possível, contudo, que, uma vez conhecendo e usando tais testes nas aulas, os alunos os retenham para futuras utilizações já no quadro da sua profissão de psicólogo, não respeitando as orientações do CFP.

Paralela a essa questão, os achados do presente artigo também mostram um número crescente de psicólogos que recorrem a testes com evidências de validade, sugerindo que estão atentos aos novos instrumentos e aos que realmente qualificam as suas avaliações. Um aprofundamento de quem são estes psicólogos pode ajudar a entender qual sua formação e que tipo de relacionamento mantêm com o CFP, podendo daí decorrer ilações sobre boas práticas a serem generalizadas.

\section{Referências}

Archer, R. P., Buffington-Vollum, J.K., Stredny, R.V., \&Handel, R.W. (2006). A survey of psychological test use patterns among forensic psychologists. Journal of Personality Assessment, 87(1), 84-94. https://dx.doi.org/10.1207/s15327752jpa8701_07

Bastos, A. V. B., \& Gomide, P. I. C. (1989). O psicólogo brasileiro: Sua atuação e formação profissional. Psicologia: Ciência e Profissão, 9(1), 6-15. https://dx.doi.org/10.1590/S1414-98931989000100003 
Bastos, A. V. B., Gondim, S. M. G., \& Borges-Andrade, J. E. (2010). O psicólogo brasileiro: Sua atuação e formação profissional. O que mudou nas últimas décadas? In O. H. Yamamoto \& A. L. F. Costa (Orgs.), Escritos sobre a profissão de psicólogo no Brasil (pp. 257-271). Natal, RN: EDUFRN.

Benson, N. F., Floydb, R. G., Kranzlerc, J. H., Eckertd, T. L., Fefere, S. A., \& Morgana, G. B. (2019). Test use and assessment practices of school psychologists in the United States: Findings from the 2017 National Survey. Journal of School Psychology, 72, 29-48. https:// doi.org/10.1016/j.jsp.2018.12.004

Bornstei, R. F. (2016). Evidence-based psychological assessment. Journal of Personality Assessment, 99(4), 435-445. https://dx.doi.org/00223891.2016.1236343

Brasil. (1962). Lei no 4.119, de 27 de agosto de 1962. Dispõe sobre a formação em Psicologia e regulamenta a profissão de psicólogo. Boletim de Psicologia, 14(44), 71-76.

Brasil. (2011). Resolução no 5, de 15 de março de 2011. Institui as Diretrizes Curriculares Nacionais para os cursos de graduação em Psicologia, estabelecendo normas para o projeto pedagógico complementar para a Formação de Professores de Psicologia. Diário Oficial da União, 1, 19.

Camara, W. J., Nathan, J. S., \& Puente, A. E. (2000). Psychological test usage: Implications in professional psychology.ProfessionalPsychology: Research andPractice,31(2), 141-154.http://dx.doi.org/ 10.1037/0735-7028.31.2.141

Conselho Federal de Psicologia. (2003). Resolução no 2/2003. Define e regulamenta o uso, a elaboração e a comercialização de testes psicológicos e revoga a Resolução CFP n²5/2001. Brasília, DF: Conselho Federal de Psicologia. http://site.cfp.org.br/wp-content/uploads/2003/03/resolucao2003_02_Anexo.pdf

Conselho Federal de Psicologia. (2018). A psicologia brasileira apresentada em números. http://www2.cfp.org.br/ infografico/quantos-somos/

Evers, A., McCormick, C., Hawley, L., Muñiz, J., Balboni, J., Bartram, . . Zhang, J. (2017). Testing practices and attitudes toward tests and testing: An international survey. International Journal of Testing, 17(2), 158-190. http://dx.doi.org:10.1080/15305058.2016.1216434

Evers, A., Muñiz, J., Bartram, D., Boben, D., Egeland, J., Fernández-Hermida, J. R., . . Urbánek, T. (2012). Testing practices in the 21st century: Developments and European psychologists' opinions. European Psychologist, 17(4), 300-319. http://dx.doi.org/10.1027/1016-9040/a000102

Hazboun, A. M., \& Alchieri, J. C. (2013). Justificativas e concepções de psicólogos que não utilizam avaliação psicológica. Avaliação Psicológica, 12(3), 361-368.

Hutz, C. S. (2009). Avanços e polêmicas em avaliação psicológica. São Paulo, SP: Casa do Psicólogo.

Hutz, C. S., \& Bandeira, D. S. (2003). Avaliação psicológica no Brasil: Situação atual e desafios para o futuro. In O. H. Yamamoto \& V. V. Gouveia (Orgs.), Construindo a Psicologia brasileira: Desafios para a ciência e prática psicológica (pp. 261-275). São Paulo, SP: Casa do Psicólogo.

Instituto Brasileiro de Opinião Pública e Estatística. (2004). Pesquisa de opinião com psicólogos inscritos no Conselho FederaldePsicologia.http://www.pol.org.br/pol/export/sites/default/pol/faq/faqDocumentos/ Pesquisa_IBOPE. pdf

Lubin, B., Larsen, R. M., \& Matarazzo, J. D. (1984). Patterns of psychological test usage in the United States: 1935-1982. American Psychologist, 39(4), 451-454. http://dx.doi.org/10.1037/0003-066X.39.4.451

Muñiz, J., \& Fernández-Hermida, J. R. (2010). La opinión de los psicólogos españoles sobre el uso de los tests. Papeles del Psicólogo, 31(1), 108-121.

Muñiz, J., Prieto, G., Almeida, L., \& Bartram, D. (1999). Test use in Spain, Portugal and Latin American countries. European Journal of Psychological Assessment, 15(2), 151-157. http://dx.doi.org/10.1027//1015-5759.15.2.151

Noronha, A. P. P. (2002). Os problemas mais graves e mais frequentes no uso dos testes psicológicos. Psicologia: Reflexão e Crítica, 15(1), 135-142. http://dx.doi.org/10.1590/S0102-79722002000100015.

Noronha, A. P. P., Primi, R., \& Alchieri, J. C. (2005). Instrumentos de avaliação mais conhecidos/utilizados por psicólogos e estudantes de psicologia. Psicologia: Reflexão e Crítica, 18(3), 390-401. https://dx.doi.org/10.1590/S0102-79722005000300013

Noronha, A. P. P., \& Reppold, C. T. (2010). Considerações sobre a avaliação psicológica no Brasil. Psicologia: Ciência e Profissão, 30, 192-201. https://dx.doi.org/10.1590/S1414-98932010000500009 
Oakland, T., Douglas, S., \& Kane, H. (2016). Top ten standardized tests used internationally with children and youth by school psychologists in 64 countries: A 24-year follow-up study. Journal of Psychoeducational Assessment, 34(2), 166-176. http://dx.doi.org/10.1177/0734282915595303

Primi, R. (2010). Avaliação psicológica no Brasil: fundamentos, situação atual e direções para o futuro. Psicologia: Teoria e Pesquisa, 26, 25-35. http://dx.doi.org/10.1590/S0102-37722010000500003

Reppold, C.T., \&Noronha, A.P.P. (2018). Impacto dos 15 Anos do Satepsi na Avaliação Psicológica Brasileira. Psicologia: Ciência e Profissão, 38(spe), 6-15. https://dx.doi.org/10.1590/1982-3703000208638

Reppold, C.T., Serafini, A. J., Gurgel, L. G., \& Kaiser,V. (2017). Avaliação de aspectos cognitivos em adultos: análise de manuais de instrumentos aprovados. Avaliação Psicológica, 16(2), 137-144. https://dx.doi.org/10.15689/AP.2017.1602.03

Reppold, C. T., Serafini, A. J., Ramires, D., \& Gurgel, L. G. (2017). Análise dos manuais psicológicos aprovados pelo SATEPSI para avaliação de crianças e adolescentes no Brasil. Avaliação Psicológica, 16(1), 19-28. https://dx.doi.org/10.15689/ap.2017.1601.03

Sundberg, N. D. (1961). The practice of psychological testing in clinical services in the United States. American Psychologist, 16(2), 79-83. http://dx.doi.org/10.1037/h0040647

World Health Organization. (2001). Pesquisa feita junto aos Associados do Conselho Federal de Psicologia: Relatório final. https://site.cfp.org.br/wp-content/uploads/2005/05/Pesquisa_WHO.pdf

\section{Caroline Tozzi Reppold}

Professora associada IV do Departamento de Psicologia, Coordenadora do Laboratório de Avaliação Psicológica/ Universidade Federal de Ciências da Saúde de Porto Alegre, Porto Alegre - RS. Brasil.

E-mail: carolinereppold@yahoo.com.br

(1) https://orcid.org/0000-0002-0236-2553

\section{Solange Muglia Wechsler}

Professora pesquisadora do curso de pós-graduação em Psicologia da Pontifícia Universidade Católica de Campinas, Campinas - SP. Brasil.

E-mail:wechsler@lexxa.com.br

(1) https://orcid.org/0000-0002-9757-9113

\section{Leandro da Silva Almeida}

Professor catedrático de Psicologia da Educação no Instituto de Educação. Investigador do Centro de Investigação em Educação da Universidade do Minho. Portugal.

E-mail: leandro@ie.uminho.pt

(1) https://orcid.org/0000-0002-0651-7014

\section{Paula Elosua}

Professor de Psicometria, Facultad de Psicología, Universidad del País Vasco, San Sebastian. Espanha.

E-mail: paula.elosua@ehu.es

(1) https://orcid.org/0000-0002-9651-9100

\section{Claudio Simon Hutz}

Professor Titular, Coordenador do Laboratório de Mensuração, da Universidade Federal do Rio Grande do Sul, Porto Alegre - RS. Brasil.

E-mail: claudio.hutz@gmail.com

(1) https:// orcid.org/0000-0002-3252-7339 
Endereço para envio de correspondência:

UFCSPA - Universidade Federal de Ciências da Saúde de Porto Alegre. Rua Sarmento Leite, 245, sala 208 - Centro Histórico. CEP 90050-170. Porto Alegre - RS. Brasil.

Recebido 29/05/2018

Aceito 30/05/2019

Received 05/29/2018

Approved 05/30/2019

Recibido 29/05/2018

Aceptado 30/05/2019

Agradecimentos:

Ao Conselho Nacional de Desenvolvimento Científico e Tecnológico (CNPq), pelo apoio científico e financiamento. Ao Instituto Brasileiro de Avaliação Psicológica (IBAP), à Associação Nacional de Pesquisa em Psicologia (ANPEPP) e ao Conselho Federal de Psicologia (CFP), pelo apoio na divulgação do levantamento. À International Test Commission (ITC) pelo apoio científico. Trabalho financiado parcialmente pelo Ministerio de Ciencia, Innovación y Universidades, da Espanha (PID2019-103859RB-I00).

Como citar: Reppold, C. T., Wechsler, S. M., Almeida, L. S., Elosua, P.. \& Hutz, C. S. (2020). Perfil dos Psicólogos Brasileiros que Utilizam Testes Psicológicos: Áreas e Instrumentos Utilizados. Psicologia: Ciência e Profissão, 40, 1-14. https://doi.org/10.1590/1982-3703003201348

How to cite: Reppold, C. T., Wechsler, S. M., Almeida, L. S., Elosua, P,. \& Hutz, C. S. (2020). Profile of Brazilian Psychologists Using Psychological Tests: Areas and Instruments Used. Psicologia: Ciência e Profissão, 40, 1-14. https://doi.org/10.1590/1982-3703003201348

Cómo citar: Reppold, C. T., Wechsler, S. M., Almeida, L. S., Elosua, P.. \& Hutz, C. S. (2020). Perfil de los Psicólogos Brasileños que Utilizan Pruebas Psicológicas: Áreas e Instrumentos Utilizados. Psicologia: Ciência e Profissão, 40, 1-14. https://doi.org/10.1590/1982-3703003201348 